\title{
Better Outcomes in High-Power Short-Duration Compared to Low-Power Long-Duration Atrial Fibrillation Ablation in One-Year Follow-Up
}

\author{
Fabricio Vassallo ${ }^{1,2,3 *}$, Lucas Luis Meigre ${ }^{1}$, Eduardo Serpa ${ }^{1,2,3}$, Carlos Lovatto ${ }^{2,3}$, Christiano \\ Cunha $^{2,3}$, Hermes Carloni ${ }^{2,3}$, Aloyr Simoes ${ }^{2,3}$, Karla Meira ${ }^{1,2}$, Flávia Pezzin ${ }^{1,2}$, Orly Lacerda ${ }^{2}$, \\ Walter Batista ${ }^{2}$, Alberto Nogueira², Dalton Amaral ${ }^{2}$ and Dalbian Gasparini ${ }^{2}$ \\ ${ }^{1}$ Instituto de Cardiologia do Espírito Santo, Brazil \\ ${ }^{2}$ Santa Rita de Cassia Hospital, Brazil
}

${ }^{3}$ Santa Casa de Misericórdia Hospital, Brazil

\begin{abstract}
Introduction: Different results are described after atrial fibrillation ablation and multiples predictors of recurrence are well established. Objective: Evaluate and analyze if patients submitted to first time atrial fibrillation (AF) ablation with high-power short-duration (HPSD) have better outcomes compared to low-power long-duration (LPLD). Methods: Observational, retrospective study, 144 patients submitted to HPSD and LPLD ablation. HPSD: 71 patients, 50(70.42\%) males, mean age 59.73 years, 52(73.24\%) hypertension, 44(61.97\%) obstructive apnea, 23(32.39\%) arterial disease, 20(28.17\%) Diabetes, and 10(14.08\%) strokes. CHADS2VASC2 2.57. CT: 73 patients, 50(68.49\%) males, mean age 60.7 years, 53(72.60\%) hypertension, 41(56.16\%) obstructive apnea, 28(38.36\%) arterial disease, $14(19.17 \%)$ Diabetes and $8(10.96 \%)$ strokes. CHAD2SVASC2 2.22. Results: Recurrence occurred in 33 patients $(22.92 \%)$ at 12 months follow-up, of these, HPSD with 9 patients and LPLD with 24 patients. At the end of study 62 (87.32\%) of 71 HPSD patients were in sinus rhythm comparing to 49 (67.12\%) of 73 patients in LPLD (P of .0039). Conclusion: HPSD compared to LPLD showed a superiority in maintaining sinus rhythm at 12 months and a significant lower recurrence rate. Also, lower procedure time, esophageal heating and radiofrequency time was significant lower in HPSD technique.
\end{abstract}

KEYWORDS: Atrial fibrillation; Atrial tachyarrhythmias; Recurrence rate; First-pass isolation; Atrial fibrillation ablation

\section{INTRODUCTION}

More than two decades ago; Haïssaguerre and his collaborators [1] concluded that ectopic beats from pulmonary veins were atrial fibrillation (AF) triggers and showed that ablation can be a treatment for these triggers. Since then, the electrophysiology community has discovered factors that limit the success of this procedure in conventional supraventricular tachycardias [2,3]. For example, as a central objective of the treatment interventions for AF; the left atrial anatomy and its surrounding organs are a limiting factor during pulmonary vein isolation (PVI) [4-7]. In addition to the anatomical limitations, questions have been raised regarding the biophysics of lesions induced by radiofrequency $[8,9]$. One
Quick Response Code:

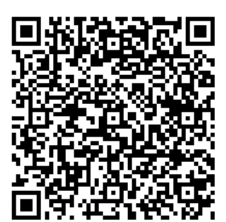

Address for correspondence: Fabricio Sarmento Vassallo MD, ElectrophysiologistCoordinator of Electrophysiology of Instituto de Cardiologia do Espírito Santo and Santa Casa de Misericórdia Hospital, Brazil

Received: July 27, $2020 \quad$ Published: September 22, 2020

How to cite this article: Fabricio V, Lucas Luis M, Eduardo S, Carlos L, Christiano C, al. et. Better Outcomes in High-Power Short-Duration Compared to Low-Power Long-Duration Atrial Fibrillation Ablation in One-Year Follow-Up. 2020 - 2(5) OAJBS.ID.000218. DOI: 10.38125/ OAJBS.000218 
of the solutions for these limitations and for the achievement of better results is the adoption of new technologies; such as irrigated catheters and; more recently; catheters containing sensors that measure real time contact force [10-14] using higher power settings in the radiofrequency generators associated with short applications times.

\section{OBJECTIVES}

The objective of the present study was to define if there is better outcome in the long-term success rate with the maintenance of sinus rhythm in patients after atrial fibrillation ablation with high- power short-duration (HPSD) compared to patients submitted to conventional ablation; with low-power long-duration (LPLD).

\section{METHODS}

\section{Study Population}

We performed 144 consecutive first catheter atrial fibrillation ablations with the LPLD and HPSD technique. This post-hoc analysis was performed with the last patients submitted to LPLD and the first one with HPSD; and data were collected between January 2016 and January 2019. Patients were divided in two groups; patients using HPSD and patients submitted to LPLD ablation (Table 1).

Table 1: Clinical features.

\begin{tabular}{|c|c|c|c|}
\hline Clinical Features & HPSD & LPLD & Statistical Analysis \\
\hline Medium Age (years) & 59.73 & 60.7 & NS \\
\hline Males (\%) & $50(70.42)$ & $50(68.49)$ & NS \\
\hline Hypertension (\%) & $52(73.24)$ & $53(72.60)$ & NS \\
\hline Obstructive Apnea (\%) & $44(61.97)$ & $41(56.16)$ & NS \\
\hline Arterial Disease (\%) & $23(32.39)$ & $28(38.34)$ & NS \\
\hline Diabetes (\%) & $20(28.17)$ & $8(10.96)$ & NS \\
\hline Stroke (\%) & $10(14.08)$ & $2.22(0-7)$ & NS \\
\hline Medium CHADS2VASC2 (Min-Max) & $2.57(0-8)$ & $52(71.23)$ & NS \\
\hline Paroxysmal AF (\%) & $39(54.93)$ & $25.63(3-144)$ & NS \\
\hline
\end{tabular}

Among the 71 HPSD patients; with mean age of 59.73 years; among them; 50 (70.42\%) were males; 52 (73.24\%) hypertension; $44(61.97 \%)$ obstructive sleep apnea; 23 (32.39\%) arterial disease; 20 (28.17\%) diabetes; and 10 (14.08\%) strokes. Mean CHADS2VASC2 2.57 (0-8). Mean left atrial diameter of $40.40 \mathrm{~mm}$ (33-62); paroxysmal AF in 39 (54.93\%) patients and the mean time from diagnosis to catheter ablation was 23 (4 to 144) months.

Among the 73 LPLD patients; with mean age of 60.7 years; among them; 50 (68.49\%) were males $53(72.60 \%)$ hypertension; 41 (56.16\%) obstructive sleep apnea; 28 (38.36\%) arterial disease; $14(19.17 \%)$ diabetes and $8(10.96 \%)$ strokes. Mean CHADS2VASC2 2.22 (0-7). Mean left atrium diameter of paroxysmal AF in 52 $(71.23 \%)$ and the mean time for AF diagnosis to ablation was in Group A and 25.63 (3-144) months.

\section{Pre-Ablation Investigation for Thrombus Exclusion}

Patients underwent a transesophageal echocardiogram (TEE) and/or angiotomography of the left atrium and pulmonary veins prior to the procedure. Both exams for exclusion of the thrombus; diameter of the left atrium; and other measurements was performed on the day of the procedure or up to 48 hours before ablation.

\section{Catheter Ablation}

All patients underwent ablation using an uninterrupted oral anticoagulation protocol (OAC). Patients also had their antiarrhythmic drugs suspended for 5 half-lives prior to the procedure; with the exception of amiodarone; which was maintained for the procedure. The main objective was to isolate the antral portion of the pulmonary veins $[2,15]$. In cases of patients with previously diagnosed atrial flutter; the tachyarrhythmia was also ablated at the end of the PVI; time for this ablation and radiofrequency were not included in the data. Procedures were performed in sinus rhythm; and patients who were in atrial fibrillation or flutter underwent electrical cardioversion immediately before the ablation. For all patients, the EnSite Velocity mapping system version 5.0 was used with a TactiCath ${ }^{\mathrm{TM}}$ contact force sensing catheter; Agilis ${ }^{\mathrm{TM}}$ deflectable sheath; Viewflex ${ }^{\mathrm{TM}}$ intracardiac echocardiography probe; and Ampere ${ }^{\mathrm{TM}}$ radio frequency generator (St Jude Medical-USA / Abbott-USA).

In the HPSD ablation technique; in the posterior wall; atrial roof; and atrial flutter (if indicated); we set the power of radiofrequency generator to 45 Watts for no more than 6 seconds. On the anterior wall, we increased the power to 50 Watts with an estimated pressure of 10-20 grams of contact force for short periods of time [16]. The irrigation pump was always programmed for $35 \mathrm{ml} / \mathrm{min}$ regardless of the power setting. For LPLD; the RF applications lasted no more than 30 seconds; we use an irrigation set of $17 \mathrm{ml} / \mathrm{min}$ for pump flow; a contact force between 10 to 30 grams and applied 20 Watts in the posterior wall and 30 Watts elsewhere including in the cases of atrial flutter. At the end of the procedure, all patients were given a challenge of $12 \mathrm{mg}$ of adenosine for each antrum of the pulmonary veins in order to unmask any dormant veins and assess the need for re-ablation of their reconnection $[17,18]$.

In addition to analyzing the real-time contact force values across all radiofrequency applications; we evaluated the impedance measurements in the EP recording system looking for gradual falls that might indicate lesion formation [8-11]. We used a dragging technique for catheter ablation (CA) and made all efforts to avoid catheter jumps. If a catheter jump did occur; the radiofrequency application was immediately interrupted; and we returned to the spot before the jump to continue the ablation [19-23]. We did not use other features provided by the contact force catheter, such as the lesion index (LSI) and force-time integral (FTI) [24]. All 
patients received esophageal temperature monitoring as described previously [25].

\section{INFORMED CONSENT AND ETHICAL CONSIDERATIONS}

All patients signed the informed consent form according to the standards of ours Institutions, which follows national and international standards $[26,27]$. The study was approved by the Research Ethics Committee of the Institution.

\section{Post-Ablation Follow-Up Protocol}

Patients who were more than 75 years old or had multiple comorbidities remained in the intensive care unit after ablation for one day; those without these characteristics stayed at an apartment and were discharged from the hospital on the day after only if clinical evaluation; vascular punctures; chest X-ray; and electrocardiogram (ECG) were normal. Patients received a prescription of Sucralfate for $2 \mathrm{~g}$ per day and Pantoprazole for $40 \mathrm{mg}$ twice daily for 4 weeks after the procedure. Same antiarrhythmic drugs were reintroduced after the procedure. Antiarrhythmics were maintained for 60 days and then suspended. Anticoagulants were interrupted at this time for patients with a CHADS2VASC2 score less than or equal to 328; except for patients who had a previous stroke and/or were 75 years old or more. In cases of atrial arrhythmia, amiodarone was the drug of choice to reestablish the normal sinus rhythm. If this approach was successful; the drug was used for 30 more days and then suspended. Direct current cardioversion (DC) was done in patients after the amiodarone challenge or if they achieved day $60^{\text {th }}$ with atrial tachyarrhythmias. All patients were evaluated after 7 days; 1 ; 2; 3; 6; and 12 months with a medical appointment and ECG. After 3; 6; and 12 months; they also underwent 24 hours of Holter monitoring.

\section{STATISTICAL ANALYSIS}

All tests were performed using BioStat statistical software (AnalystSoft Walnut; CA; USA). Continuous variables were expressed as mean-standard deviation. A result was considered significant if $\mathrm{P}$ value of $<.05$. The continuous variables were compared using the qui-square test were also used.

\section{RESULTS}

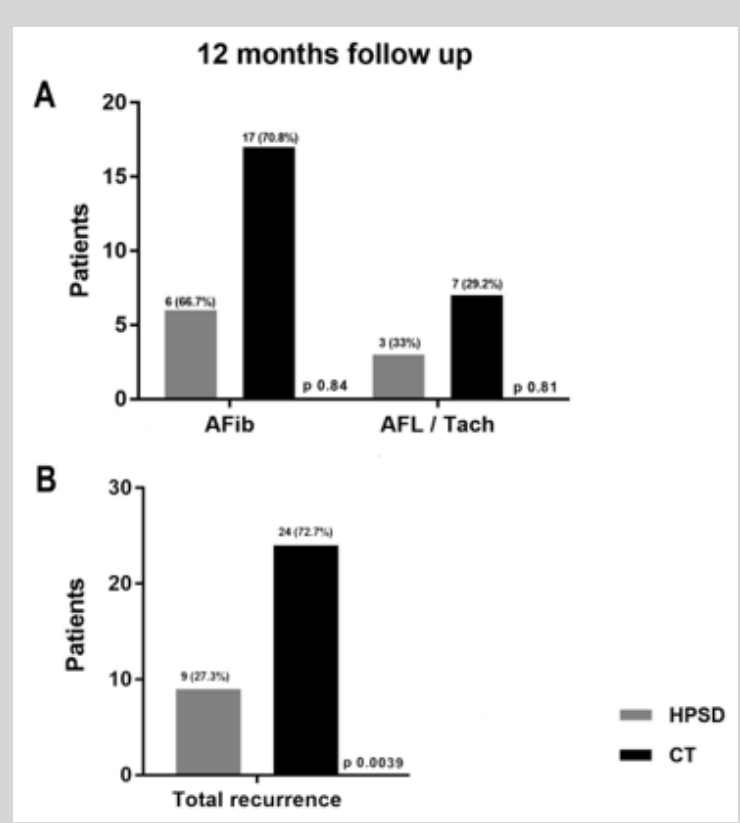

Figure 1: Types of atrial tachyarrhythmias recurrences at the top and the total recurrence rate at the bottom in HPSD and CT (LPLD) ablations.

In the 12 months analysis; $62(87.32 \%)$ of 71 HPSD patients were in sinus rhythm comparing to $49(67.12 \%)$ of 73 patients in LPLD; demonstrating a significant $P$ value of .0039; showing that when treated with the first approach patients had better outcomes with a much lower rate of recurrences (Figure 1). Of the 9 recurrent patients in HPSD ablation $6(66.67 \%)$ were in AF and
$3(33.33 \%)$ in atypical atrial flutter/tachycardia; while in the LPLD patients 17 (70.83\%) were in AF and only 7 (29.17\%) in regular tachyarrhythmias; with non-significant $P$ values (Table 2). In our study we only evaluate patients in first time ablation and a redo procedure was not performed in this group of patients.

Table 2: Recurrence characteristics at 12 Months in HPSD and CT (Conventional technique with low-power long duration) patients.

\begin{tabular}{|c|c|c|c|}
\hline Recurrence Rate 12 months & HPSD & LPLD & Statistical Analysis \\
\hline $\begin{array}{c}\text { Atrial Arrhythmias at ECG and/or Holter } \\
\text { (Total: 33 pts) }\end{array}$ & $9(27.27 \%)$ & $24(72.73 \%)$ & 0.0039 \\
\hline Atrial Fibrillation & $6(66.67 \%)$ & $17(70.83 \%)$ & 0.84 \\
\hline Atrial Flutter / Tachycardia & $3(33.33 \%)$ & $7(29.17 \%)$ & 0.81 \\
\hline
\end{tabular}


Making more data analysis of other findings of this study we observed some differences in the results between the two techniques: (1) lower rate of esophageal heating in HPSD with 34 of 71 patients showing elevation in esophageal temperature in comparison to 52 of 73 patients in LPLD with a significant P-value of .0019; (2) equal left atrium time between the techniques; with a P value of.0839; (3) lower total ablation time; with 94.54 minutes in HPSD and 113.55 minutes in LPLD achieving a significant P-value of .0059; (4) a major difference in the radiofrequency time (RF); with
HPSD protocol showing 1964.84 seconds and LPLD with 5017.80 seconds; and a remarkable P-value of $<0.00001$ (Table 3). Another interesting finding of our date was a small number of reconnections spontaneously or after a challenge of $12 \mathrm{mg}$ of Adenosine. In this series of patients; HPSD ablation showed a total of $4(5.63 \%)$ left PVs reconnections and $3(4.23 \%)$ in right PVs and LPLD ablation; $5(6.85 \%)$ in left PVs and $3(4.11 \%)$ in right PVs; with a nonsignificant P-value.

Table 3: Ablation features in HPSD and CT (Conventional technique with low-power long duration). NS= non-significant with $P$ value of $>.05$

\begin{tabular}{|c|c|c|c|}
\hline Ablation Features & HPSD 71 patients & LPLD 73 patients & Statistical Analysis \\
\hline Mean Left Atrial time (min) & 76.16 & 92.79 & NS \\
\hline Mean Total ablation time (min) & 96.54 & 113.55 & 0.0059 \\
\hline Mean 3D Map Collecting Time (min) & 8.32 & 7.28 & NS \\
\hline Mean RF Time (sec) & 1961.25 & $5,017,80$ & $<.00001$ \\
\hline Mean Fluoroscopy Time (min) & 7.6 & 13.37 & NS \\
\hline Esophageal Heating & $34(47.89 \%)$ & $49(71.23 \%)$ & 0.0019 \\
\hline $\begin{array}{c}\text { Success rate (sinus rhythm at 12 } \\
\text { months follow-up) }\end{array}$ & $62(87.32 \%)$ & $49.12 \%)$ & 0.0039 \\
\hline
\end{tabular}

\section{DISCUSSION}

In this retrospective; observational study; we observed a high rate of sinus rhythm at 12 months follow-up in patients submitted to HPSD compared to those submitted to LPLD ablation. In our first report16 comparing these two techniques last year; in 76 patients we do not saw a clear benefit in terms of better outcome in maintenance of sinus rhythm comparing the two groups. With a recurrence rate of $17.07 \%$ in the HPSD group and $31.42 \%$ in LPLD the $P$ value was .14 but we observed that this could be a bias and if we follow these patients for a longer time or compare a higher number of patients in both groups result could be distinct favoring a better outcome for HPSD patients [28].

The first report from our knowledge was that from Bunch et al. [29] also comparing the results for long term outcome; with 3 years of follow-up; showed no superiority of the HPSD ablation compared to the LPLD approach. The major difference between both studies was that in all of our patients the ablation was performed with a contact force sensing catheter and this was not observed in their patients. Since this; we try to perform a study including only patients submitted to AF ablation with contact force sensing catheters; which is the subject of this manuscript.

As in another series of cases we had a small number of reconnections spontaneously or after a challenge of $12 \mathrm{mg}$ of Adenosine. In our series; in HPSD ablation we had a total of 4 (5.63\%) left PVs reconnections and 3 (4.23\%) in right PVs and in LPLD ablation $5(6.85 \%)$ in left PVs and $3(4.11 \%)$ in right PVs: with a non-significant $P$ value. In the paper of Kamatsu et al. [30] that in 20 patients submitted to HPSD ablation they had no cases of reconnection; and in the paper by Berte et al. [31]; the rate of reconnections in two different groups with different approaches varied from 21 to 33\%. In these two studies using HPSD ablation the rate of PVs reconnections varied from 33 to $0 \%$ showing a huge variation in this type of technique.

As a matter of additional information, 4 HPSD patients went back to the electrophysiology lab for a redo procedure since they had symptomatic tachyarrhythmias that failed to medical therapy; 2 patients were in AF and 2 in atypical atrial flutter. The electrophysiologic findings was 3 patients without PVs reconnection and 1 patient with the left PVs reconnected. Of the LPLD patients, 16 of the 24 recurrent patients were submitted to a redo procedure with 11 showing $\mathrm{AF}$ and 5 atypical atrial flutters. Of these 16; in 12 patients at least $1 \mathrm{PV}$ was reconnected and in the other 4 patients we did not document any PV reconnection; with the ablation procedure directed to treat left atrial flutters. Our results suggest; in a retrospective analysis of theses 144 patients; that patients submitted to HPSD ablation had at 12 months $87.32 \%$ of patients in sinus rhythm; while in LPLD only $67.12 \%$.

\section{STUDY LIMITATIONS}

This was a retrospective; observational; small sampled size study that practiced non-continuous cardiac rhythm monitoring using ECGs and 24-hour Holter monitoring to document the recurrence of atrial tachyarrhythmias. A larger sample size or continuous monitoring may produce in future different findings.

\section{CONCLUSION}

This study showed a high success rate of sinus rhythm maintenance for HPSD compared to LPLD with significant lower recurrence rates for patients treated with the first technique. Besides this result patients in HPSD ablation had significant lower procedure times, lower esophageal heating and radiofrequency times.

\section{ACKNOWLEDGMENT}

For their dedication and effort in data collection and patience; we appreciate the dedication of Christiane Amaral and Vanessa Rodrigues from the administration staff and Edevaldo da Silva; Marcel Burguês; and Rafael Zeni from the biomedical team.

\section{REFERENCES}

1. Haïssaguerre M, Jaïs P, Shah DP, Takahashi A, Hocini M, et al. (1998) Spontaneous initiation of atrial fibrillation by ectopic beats originating in the pulmonary veins. N Engl J Med 339(10): 659-666. 
2. Cappato R, Calkins H, Chen SA, Davies W, Iesaka Y, et al. (2010) Updated worldwide survey on the methods, efficacy, and safety of catheter ablation for human atrial fibrillation. Circ Arrhythm Electrophysiol 3(1): 32-38.

3. Calkins H, Brugada J, Packer DL, Cappato R, Chen SA, et al. (2007) HRS/ EHRA/ECAS expert consensus statement on catheter and surgical ablation of atrial fibrillation: recommendations for personnel, policy, procedures and follow-up. A report of the Heart Rhythm Society (HRS) Task Force on Catheter and Surgical Ablation of Atrial Fibrillation developed in partnership with the European Heart Rhythm Association (EHRA) and the European Cardiac Arrhythmia Society (ECAS). Europace. 9(6): 335-379.

4. Oral H, Knight BP, Tada H, Ozaydin M, Chugh A, et al. (2002) Pulmonary vein isolation for paroxysmal and persistent atrial fibrillation. Circulation 105(9): 1077-1081.

5. Ho SY, Sanchez-Quintana MD, Cabrera JA, Anderson RH (1999) Anatomy of the left atrium: implications for radiofrequency ablation of atrial fibrillation. J Cardiovasc Electrophysiol 10(11): 1525-1533.

6. Sánchez-Quintana D, Cabrera JA, Climent V, Farré J, Mendonça MC, et al. (2005) Anatomic relations between the esophagus and left atrium and relevance for ablation of atrial fibrillation. Circulation 112(10): 14001405.

7. Ho SY, Cabrera JA, Sanchez-Quintana D (2012) Left atrial anatomy revisited. Circ Arrhythm Electrophysiol 5(1): 220-228.

8. Haines DE (1993) The biophysics of radiofrequency catheter ablation in the heart: the importance of temperature monitoring. PACE. 16(2): 586-591.

9. Panescu D, Whayne JG, Fleischman SD, Mirotznik MS, Swanson DK, et al. (1995) Three-dimensional finite element analysis of current density and temperature distributions during radio-frequency ablation. IEEE Transactions on Biomedical Engineering 42(9): 879-890.

10. Nakagawa H, Wittkampf FHM, Yamanashi WS, Pitha JV, Imai S, et al. (1998) Inverse relationship between electrode size and lesion size during radiofrequency ablation with active electrode cooling. Circulation 98(5): 458-465.

11. Bruce GK, Bunch TJ, Milton MA, Sarabanda A, Johnson SB, et al. (2005) Discrepancies between catheter tip and tissue temperature in cooledtip ablation: relevance to guiding left atrial ablation. Circulation 112(7): 954-960.

12. Guy DJR, Boyd A, Thomas SP, Ross DL (2003) Increasing power versus duration for radiofrequency ablation with a high superfusate flow: implications for pulmonary vein ablation? PACE 26(6): 1379-1385.

13. Ullah W, McLean A, Tayebjee MH, Gupta D, Ginks MR, et al. (2016) Randomized trial comparing pulmonary vein isolation using the SmartTouch catheter with or without real-time contact force data. Heart Rhythm 13(9): 1761-1767.

14. Kautzner J, Neuzil P, Lambert H, Peichl P, Petru J, et al. (2015) EFFICAS II: optimization of catheter contact force improves outcome of pulmonary vein isolation for paroxysmal atrial fibrillation. Europace 17(8): 12291235.

15. Vassallo F, Cunha C, Serpa E, Meigre LL, Carloni H, et al. (2019) Comparison of high-power short-duration (HPSD) ablation of atrial fibrillation using a contact force-sensing catheter and conventional technique: Initial results. J Cardiovasc Electrophysiol 10:1877-1883.

16. Saad E, D’Avila A, Costa IP, Aryana A, Slater C, et al. (2011) Very low risk of thromboembolic events in patients undergoing successful catheter ablation of atrial fibrillation with a CHADS2 score $\leq 3$. A long-term outcome. Circ Arrhythm Electrophysiol 4(5): 615-621.

17. McLellan AJA, Kumar S, Smith C, Morton JB, Kalman JM, (2013) The role of adenosine following pulmonary vein isolation in patients undergoing catheter ablation for atrial fibrillation: a systematic review. J Cardiovasc Electrophysiol 24(7): 742-751
18. Macle L, Khairy P, Weerasooriya R, Novak P, Verma A, et al. (2015) Adenosine-guided pulmonary vein isolation for the treatment of paroxysmal atrial fibrillation: an international, multicentre, randomised superiority trial. Lancet 386(9994): 672-679.

19. Kuck KH, Reddy VY, Schmidt B, Natale A, Neuzil P, et al. (2012) A novel radiofrequency ablation catheter using contact force sensing: Toccata study. Heart Rhythm 9(1): 18-23.

20. Reddy VY, Shah D, Kautzner J, Schmidt B, Saoudi N, et al. (2012) The relationship between contact force and clinical outcome during radiofrequency catheter ablation of atrial fibrillation in the TOCCATA study. Heart Rhythm 9(11): 1789-1795

21. Yokokawa M, Bhandari AK, Tada H, Suzuki A, Kawamura M, et al. (2011) Comparison of the point-by-point versus catheter dragging technique for curative radiofrequency ablation of atrial fibrillation. PACE 34(1): $15-22$

22. Neuzil P, Reddy VY, Kautzner J, Petru J, Wichterle D, et al. (2013) Electrical reconnection after pulmonary vein isolation is contingent on contact force during initial treatment results from the EFFICAS I study. Circ Arrhythm Electrophysiol 6(2): 327-333.

23. Kautzner J, Peichl P (2014) Contact force assessment in catheter ablation of atrial fibrillation. J Atr Fibrillation 6(6): 1047.

24. Kautzner J, Neuzil P, Peichl P, Petru J, Cihak R, et al. (2012) EFFICAS II: optimization of catheter contact force improves outcome of pulmonary vein isolation for paroxysmal atrial fibrillation. Heart Rhythm 17(8): 1299-1235.

25. Leite LR, Santos SN, Maia H, Henz BD, Giuseppin F, et al. (2011) Luminal esophageal temperature monitoring with a deflectable esophageal temperature probe and intracardiac echocardiography may reduce esophageal injury during atrial fibrillation ablation procedures: results of a pilot study. Circ Arrhythm Electrophysiol 4(2): 149-156.

26. Ministry of Health (2015) MS National Agency of Sanitary Surveillance. Anvisa Resolution of the Board of Directors.

27. Rickham PP (1964) Human experimentation: code of ethics of the World Medical Association: declaration of Helsinki. Br Med J 2: 177.

28. Bunch TJ, May HT, Bair TL, Crandall BG, Cutler MJ, et al. (2020) Longterm outcomes after low power, slower movement versus high power, faster movement irrigated-tip catheter ablation for atrial fibrillation. Heart Rhythm 17: 184-189.

29. Okamatsu H, Koyama J, Sakai Y, Negishi K, Hayashi K, et al. (2019) Highpower application is associated with shorter procedure time and higher rate of first-pass pulmonary vein isolation inablation index-guided atrial fibrillation ablation. J Cardiovasc Electrophysiol 30(12): 1-8.

30. Berte B, Hilfiker G, Moccetti F, Schefer T, Weberndörfer V, et al. (2019) Pulmonary vein isolation using ablation index vs. CLOSE protocol with a surround flow ablation catheter. Europace 22(1): 1-6.

31. Calkins H, Kuck KH, Cappato R, Brugada J, Camm AJ, Chen SA, et al (2012) HRS/EHRA/ECAS expert consensus statement on catheter and surgical ablation of atrial fibrillation: recommendations for patient selection, procedural techniques, patient management and follow-up, definitions, end points, and research trial design: a report of the Heart Rhythm Society (HRS) Task Force on Catheter and Surgical Ablation of Atrial Fibrillation. Developed in partnership with the European Heart Rhythm Association (EHRA), a registered branch of the European Society of Cardiology (ESC) and the European Cardiac Arrhythmia Society (ECAS); and in collaboration with the American College of Cardiology (ACC), American Heart Association (AHA), the Asia Pacific Heart Rhythm Society (APHRS), and the Society of Thoracic Surgeons (STS). Endorsed by the governing bodies of the American College of Cardiology Foundation, the American Heart Association, the European Cardiac Arrhythmia Society, the European Heart Rhythm Association, the Society of Thoracic Surgeons, the Asia Pacific Heart Rhythm Society, and the Heart Rhythm Society. Heart Rhythm 9: 632-696. 